\title{
Inhibition of cartilage breakdown by hydrocortisone in a tissue culture model of rheumatoid arthritis
}

\author{
JAMES J. STEINBERG, *STUART B. KINCAID, AND CLEMENT B. SLEDGE \\ From the Orthopaedic Research Laboratory, Robert B. Brigham Division, Departments of *Medicine and \\ Orthopaedic Surgery, Brigham and Women's Hospital, Harvard Medical School, Boston, Massachusetts \\ 02115, USA
}

SUMMARY Bovine nasal cartilage discs cocultured with human rheumatoid synovial membrane or synovial-membrane-conditioned media release proteoglycan largely as a result of cartilage breakdown. We assessed the effects of hydrocortisone on proteoglycan distribution between cartilage and culture medium, and on cartilage breakdown expressed as the release of either proteoglycan or ${ }^{35} \mathrm{~S}$-products from prelabelled discs. The presence of synovial membrane inhibited the capacity for net proteoglycan synthesis, preventing its accumulation in cartilage; this was little affected by hydrocortisone. The major response to pharmacological concentrations of hydrocortisone was suppression of both spontaneous and synovial-membrane-induced cartilage breakdown. The autolysis of synovial protein that normally occurred during culture was similarly prevented by comparable doses of corticosteroid. Changes in chromatographic distribution of the ${ }^{35} \mathrm{~S}$-labelled degradation products released from cartilage conformed with a corticosteroid-induced inhibition of endogenous lysosomal or related proteinase activity. Additionally, inhibition of the early events in synovial membrane that are responsible for chondrocyte-mediated breakdown of cartilage may contribute significantly to the overall corticosteroid effect.

The destruction of articular cartilage in human rheumatoid arthritis results from the concerted actions of a number of degradative enzymes found in one or more cellular elements of the diseased joint. ${ }^{2}$ The invasive properties of rheumatoid pannus ${ }^{23}$ clearly account for much of the breakdown commonly seen. In addition mounting evidence points to a second mechanism independent of direct contact in which the chemically mediated breakdown of cartilage results from an interaction with synovial membrane that requires the participation of living chondrocytes. $^{4-7}$

We have previously described a model system that reproduces in organ culture the confrontation of synovial membrane and cartilage occurring in the rheumatoid joint, and we have characterised the quantitative aspects of proteoglycan breakdown ${ }^{6}$ and its response to antirheumatic drugs. ${ }^{8}$ Pharmacological levels of hydrocortisone in-vitro effectively suppress both spontaneous and synovial-membrane-stimulated cartilage breakdown in this system. Each of these 2 components is inhibited independently and in

Accepted for publication 14 May 1982.

Correspondence to J. J. Steinberg, MD, Orthopaedic Research Laboratory, Brigham and Women's Hospital, 75 Francis Street, Boston, MA 02115, USA direct proportion to its initial magnitude. ${ }^{8}$ In the present report we characterise the inhibitory response to hydrocortisone and the nature of the proteoglycan breakdown products released in culture. The principal hydrocortisone response is consistent with a limiting effect of the drug on the breakdown of proteoglycan core-protein, attributable to reduced lysosomal proteinase activity.

\section{Materials and methods}

CULTURES

Uniform discs $(1 \times 8 \mathrm{~mm})$ of bovine nasal cartilage were prepared as described previously. ${ }^{6}$ A sufficient number was prepared in each experiment to provide at least 5 replicates for each group of cultures. Discs established as individual organ cultures were fed at 2-day intervals by complete change of culture medium (Dulbecco's modified Eagle's medium-high glucose, supplemented with $10 \%(v / v)$ fetal bovine serum, 100 units penicillin/ml, $100 \mu \mathrm{g}$ streptomy$\mathrm{cin} / \mathrm{ml}$, and $5 \mu \mathrm{g}$ amphotericin-B/ml). In some experiments cartilage discs were first labelled with ${ }^{35} \mathrm{SO}_{4}{ }^{2-}$ by incubation in low-sulphate $\left(\mathrm{MgCl}_{2}\right.$ substituted for $\mathrm{MgSO}_{4}$ ) medium containing $10 \mu \mathrm{Ci} / \mathrm{ml}$ carrier-free $\mathrm{Na}_{2}{ }^{35} \mathrm{SO}_{4}$ (New England 
Nuclear Corp.). After $24 \mathrm{~h}$ incubation with constant agitation on a roller apparatus radioactive medium was decanted, discs were washed with several changes of phosphate-buffered saline, and then established as separate organ cultures in sulphatereplete 'chase' medium. Incubation was continued for a further $48 \mathrm{~h}$ before adding synovial membrane. We have shown that all ${ }^{35} \mathrm{~S}$-radioactivity in discs prepared in this fashion is incorporated in cartilage macromolecules. ${ }^{9}$

Human rheumatoid synovial membranes obtained fresh at the time of reconstructive joint surgery were stripped of adherent tissue, washed, and minced. Equal portions delivered from a wide-bore pipette were applied to cartilage discs, overlapping the edges by a few millimeters. Cultures were incubated in the presence or absence of hydrocortisone hemisuccinate (Solu-Cortef) for up to 8 days, and media were harvested at 2-day intervals. Portions of synovial membrane and several cartilage discs set aside as timezero samples, and cartilage discs plus synovial tissue recovered at the end of each experiment, were all stored at $-20^{\circ} \mathrm{C}$ for analysis.

In some experiments synovial-membrane-conditioned media were prepared in the absence of cartilage. Minced portions of rheumatoid synovial membrane were incubated in complete medium (10 $\mathrm{ml} / \mathrm{g}$ tissue) with or without hydrocortisone, in $75 \mathrm{~cm}^{2}$ tissue culture flasks (Falcon no. 3024) gently oscillating on a rotatory shaker in $\mathrm{CO}_{2}$ : air, 1:19. The initial overnight medium was discarded; subsequent 2-day medium changes were harvested for up to 8 days, sedimented at $800 \mathrm{~g}$ for $10 \mathrm{~min}$, and held at $-20^{\circ} \mathrm{C}$ for assay.The presence of synovial factor(s) that induce cartilage degradation was assayed by comparing the proteoglycan released by live discs versus discs killed by repeated freezing-thawing. Assay discs were cultured for 8 days either in synovialmembrane-conditioned media diluted with serumfree fresh medium (1:1) or in complete fresh medium containing $5 \%(\mathrm{v} / \mathrm{v})$ fetal bovine serum.

ANALYTICAL METHODS

${ }^{35} \mathrm{~S}$-Labelled products released into culture medium were fractionated on a column $(1.5 \times 28.5 \mathrm{~cm})$ of Sepharose 2B (Pharmacia Fine Chemicals) eluted with $0.15 \mathrm{M} \mathrm{NaCl}, 0.02 \mathrm{M}$ sodium acetate buffer $(\mathrm{pH}$ $5 \cdot 8), 0.02 \% \mathrm{NaN}_{3}$ at a flow rate of $8 \mathrm{ml} / \mathrm{h}$. The column was calibrated with blue dextran $2000\left(\mathrm{~V}_{0}\right)$, chondroitin sulphate $\left(\mathrm{V}_{\mathrm{cs}}\right)$ and ${ }^{35} \mathrm{SO}_{4}{ }^{2-}\left(\mathrm{V}_{\mathrm{t}}\right)$. Native ${ }^{35} \mathrm{~S}$-proteoglycan extracted from labelled cartilage discs by the method of Sajdera and Hascall ${ }^{10}$ and purified by associative sedimentation in a $\mathrm{CsC} 1$ gradient were eluted almost entirely in the void volume $\left(\mathrm{V}_{0}\right)$ of this column. Labelled proteoglycan products of smaller hydrodynamic size, such as those released in synovial-membrane-cartilage cocultures (see below), were distinguished from higher-order aggregates by a broader range of elution volumes $\left(\mathrm{V}_{\mathrm{e}}\right)$, their distribution being defined by an average partition coefficient $\mathrm{K}_{\mathrm{av}}=\left(\mathrm{V}_{\mathrm{e}}-\mathrm{V}_{\mathrm{o}}\right) /\left(\mathrm{V}_{\mathrm{t}}=\mathrm{V}_{\mathrm{o}}\right)$.

For synovial protein determination samples were dissolved in concentrated formic acid $\left(65^{\circ} \mathrm{C}, 16 \mathrm{~h}\right)$. Aliquots dried in vacuo were hydrolysed in $6 \mathrm{M} \mathrm{HCl}$ $\left(110^{\circ} \mathrm{C}, 16 \mathrm{~h}\right)$ and analysed by the ninhydrin reaction. ${ }^{11}$ Cartilage discs were solubilised by two $24 \mathrm{~h}$ digestions with papain as described previously. ${ }^{6}$ Proteoglycan content expressed as chondroitin sulphate equivalents was determined by the modified $^{6}$ alcian blue complexing method. ${ }^{12}$ Radioactivity was counted in a Packard Tricarb liquid scintillation spectrometer (model no. 3375) and values were corrected for quenching by external standardisation. Where appropriate, mean data were compared by the standard $t$ test. ${ }^{13}$

\section{Results}

PROTEOGLYCAN RELEASE AS A FUNCTION OF SYNOVIAL DOSE

Volumetric delivery of the synovial suspension provided good replication of the synovial dose. In a preliminary survey ninhydrin assay of 65 samples $(13$ groups, 5 cultures each) agreed with a coefficient of

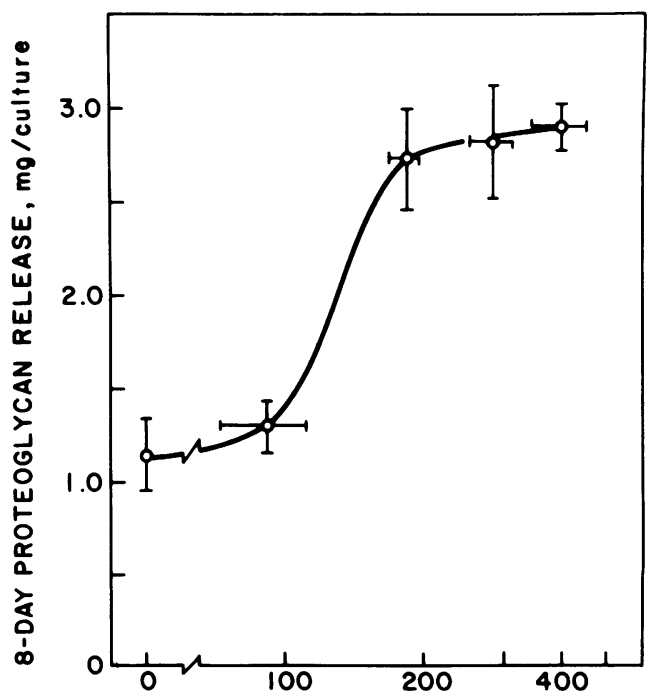

SYNOVIAL PROTEIN, $\mu$ mol amino acid/culture

Fig. 1 Cartilage breakdown as a function of synovial dose. Minced synovial membrane in suspension was applied to replicate cartilage discs at volumes of $0 \cdot 25,0 \cdot 5$ (the standard volume, here corresponding to $184 \mu \mathrm{mol}$ amino acid). 1 , and $2 \mathrm{ml}$. Proteoglycan released into culture medium during 8 days was compared to the release from control discs incubated alone. Synovial specimens were recovered at the end of culture. Values are means \pm SEM of 5 cultures. 
variation (SD/mean) of $<20 \%$. The dependence of proteoglycan release upon synovial dose is demonstrated in Fig. 1. In this experiment multiples of a standard volume $(0.5 \mathrm{ml})$ were applied to different groups of discs. Although strikingly dose-dependent at low levels, proteoglycan release reached a plateau at a synovial protein content equivalent to $200 \mu \mathrm{mol}$ of amino acid per culture. To ensure that synovial dose would not be limiting, this quantity of protein was delivered in all subsequent experiments.

HYDROCORTISONE DOSE RESPONSE

Synovial protein recovered at the end of 8 days' culture (either alone or in the presence of cartilage) was noted frequently to be less than the amount originally applied. As illustrated in Fig. 2A, synovial protein content was depleted by $38 \%$ when cultured in the absence of hydrocortisone. Preservation of protein improved at progessively higher hydrocortisone concentrations, so that at $10 \mu \mathrm{g}$ hydrocortisone $/ \mathrm{ml}$ the values at 8 days were indistinguishable from starting levels.

Cartilage breakdown in cocultures also varied as a function of hydrocortisone concentration and was reciprocally related to the change in synovial protein content. In the absence of hydrocortisone proteoglycan release was enhanced to $1 \cdot 43$-fold the basal level (Fig. 2B); this was significantly inhibited at pharmacological concentrations $(>1 \mu \mathrm{g} / \mathrm{ml})$ similar to those at which synovial protein loss was minimised or abolished (Fig. 2A). In the absence of synovial membrane spontaneous proteoglycan release was reduced only at $10 \mu \mathrm{g}$ hydrocortisone $/ \mathrm{ml}$. Synovial membrane itself, despite the loss of its protein, contributed negligible proteoglycan throughout, whether hydrocortisone was present or not.

\section{PROTEOGLYCAN DISTRIBUTION}

The inhibitory effect of hydrocortisone was usually detectable within 4 days. At the end of 8 days cumulative proteoglycan release into the medium of cartilage-synovial-membrane cocultures was reduced by an average of $50 \%$, while control cartilages showed only a modest $15 \%$ response. ${ }^{8}$ We have previously shown that the greater part of medium proteoglycan results from cartilage breakdown, ${ }^{6}$ and that its two components-i.e., spontaneous release and breakdown stimulated by synovial membrane-are individually responsive to corticosteroid inhibition. ${ }^{8}$ In the experiment illustrated in Fig. 3 the overall distribution of proteoglycan and its response to hydrocortisone after 8 days' culture are illustrated. In all cultures there was a significant increase in total proteoglycan content, indicating ongoing net synthesis. In the absence of hydrocortisone, synovial membrane inhibited proteoglycan synthesis $(\mathrm{C}+\mathrm{S}-\mathrm{H}$ versus $\mathrm{C}-\mathrm{H}, \mathrm{p}<0.02)$, sharply reducing its deposition in cartilage $(\mathrm{p}<0.001)$ and stimulating its release into culture medium, where it comprised $58 \%$ of the total $(\mathrm{C}+\mathrm{S}-\mathrm{H}$ versus $\mathrm{C}-\mathrm{H}, \mathrm{p}<0.05)$. The addition of hydrocortisone to such cocultures $(\mathrm{C}+\mathrm{S}+\mathrm{H})$ produced only minor additional inhibition of synthesis, but significantly reduced the fraction of proteoglycan

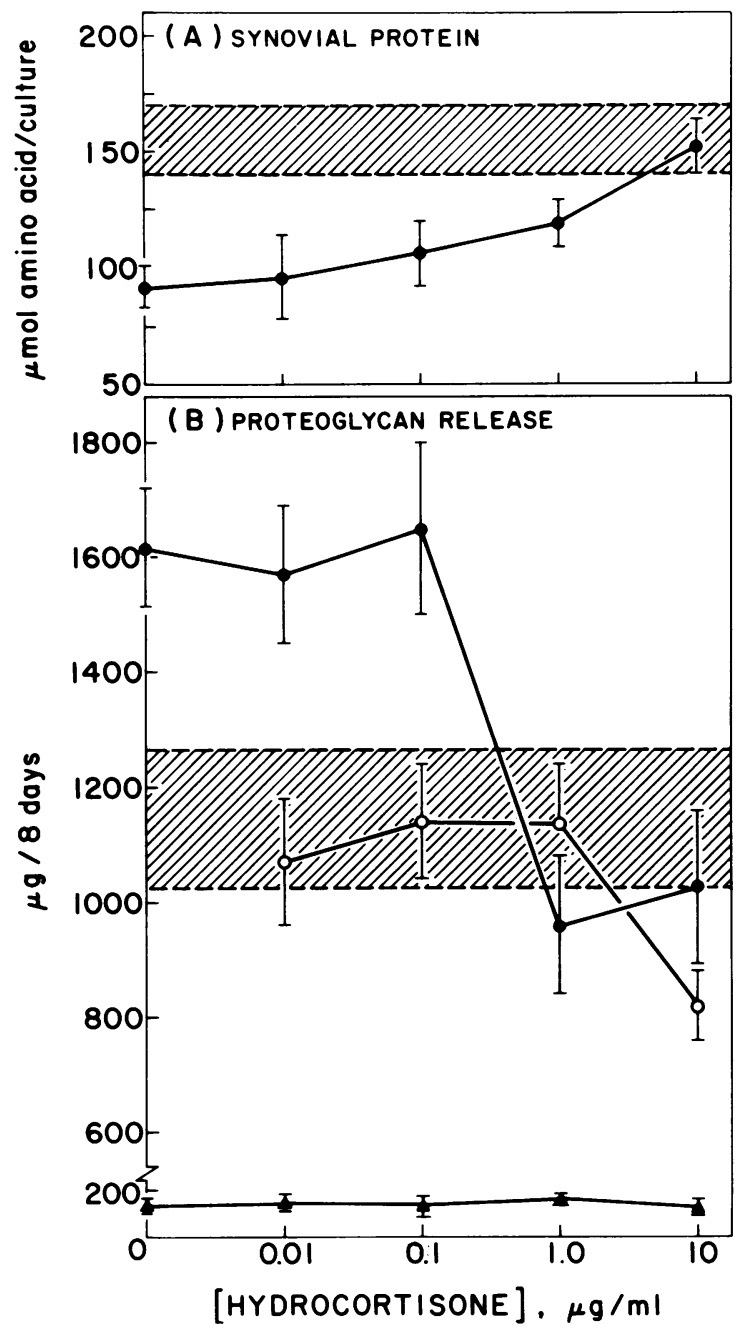

Fig. 2 Inhibition of cartilage and synovial breakdown by hydrocortisone. Cultures were incubated for intervals up to 8 days in the presence of hydrocortisone at the indicated concentrations. Values are means $\pm S E M$ of 5-9 cultures. (A) The protein content of replicate synovial specimens recovered at the end of culture $(O)$ is compared to the content at time-zero (shaded area, mean $\pm S E M)$. (B)Proteoglycan release from cultures containing cartilage $(O)$, cartilage plus synovial membrane $(\mathbf{O})$, or synovial membrane alone $(\Delta)$ is compared with the 8-day release in basal cultures containing cartilage alone with no added hydrocortisone (shaded area, mean $\pm S E M$ ). 


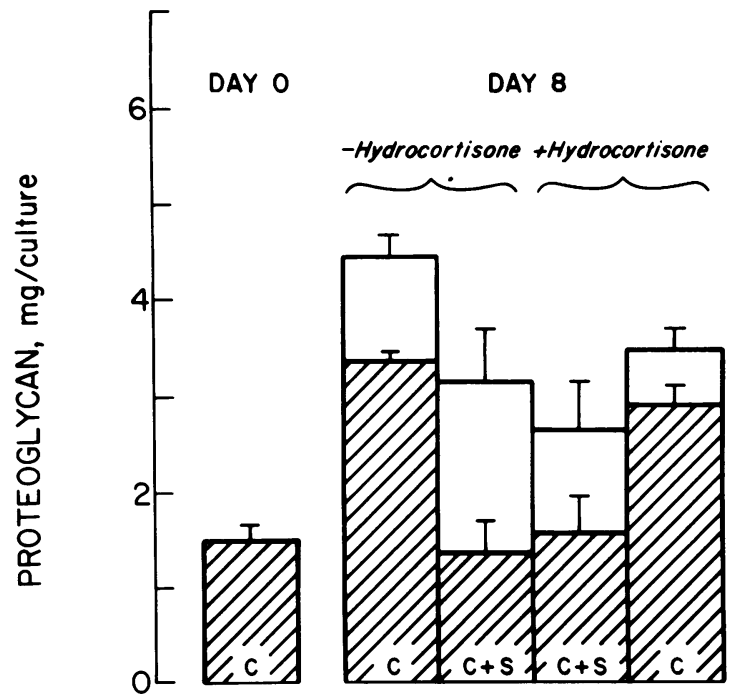

Fig. 3 Effect of hydrocortisone on proteoglycan content and distribution in culture. Five cultures in each group were incubated in the absence or the presence of hydrocortisone $(10 \mu \mathrm{g} / \mathrm{ml})$ for 8 days. Proteoglycan accumulating in the

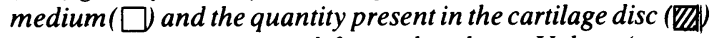
at that time were summed for each culture. Values (means $\pm S E M)$ are compared with the cartilage proteoglycan content at the outset of culture. $C=$ cartilage alone, $C+S=$ cartilage plus synovial membrane.

in the culture medium to $40 \%$ of the total $(\mathrm{C}+\mathrm{S}+\mathrm{H}$ versus $\mathrm{C}+\mathrm{S}-\mathrm{H}, \mathrm{p}<0.02)$ In absence of synovial membrane hydrocortisone could be shown to inhibit overall synthesis $(\mathrm{C}+\mathrm{H}$ versus $\mathrm{C}-\mathrm{H}, \mathrm{p}<0.025)$, but the most striking effect was on breakdown, with a reduction of medium proteoglycan to $16 \%$ of the total $(\mathrm{C}+\mathrm{H}$ versus $\mathrm{C}-\mathrm{H}, \mathrm{p}<0.05)$. Thus synovial membrane inhibited proteoglycan accumulation, curtailing its deposition in cartilage on the one hand and stimulating its breakdown on the other. Hydrocortisone effectively inhibited both spontaneous and synovial-membrane-induced cartilage breakdown with only minor effects on overall proteoglycan synthesis.

BREAKDOWN OF PRELABELLED CARTILAGE We have calculated elsewhere that perhaps as much as one-third of medium proteoglycan arises from sources other than cartilage degradation-for example metabolic 'shunting'. ${ }^{6}$ Thus to assess the hydrocortisone effect specifically on breakdown, cultures were established with ${ }^{35} \mathrm{~S}$-prelabelled cartilage discs, in which all radioactivity is initially in cartilage macromolecules, ${ }^{9}$ and therefore radioactivity subsequently released into culture medium unequivocally arises from cartilage breakdown. The experiment shown in Fig. 4 illustrates the time course of proteo- glycan breakdown in such prelabelled cultures. The rate of proteoglycan release (Fig. 4A) was stimulated several fold in synovial-membrane-containing cultures. This generally occurred after an initial 2-day

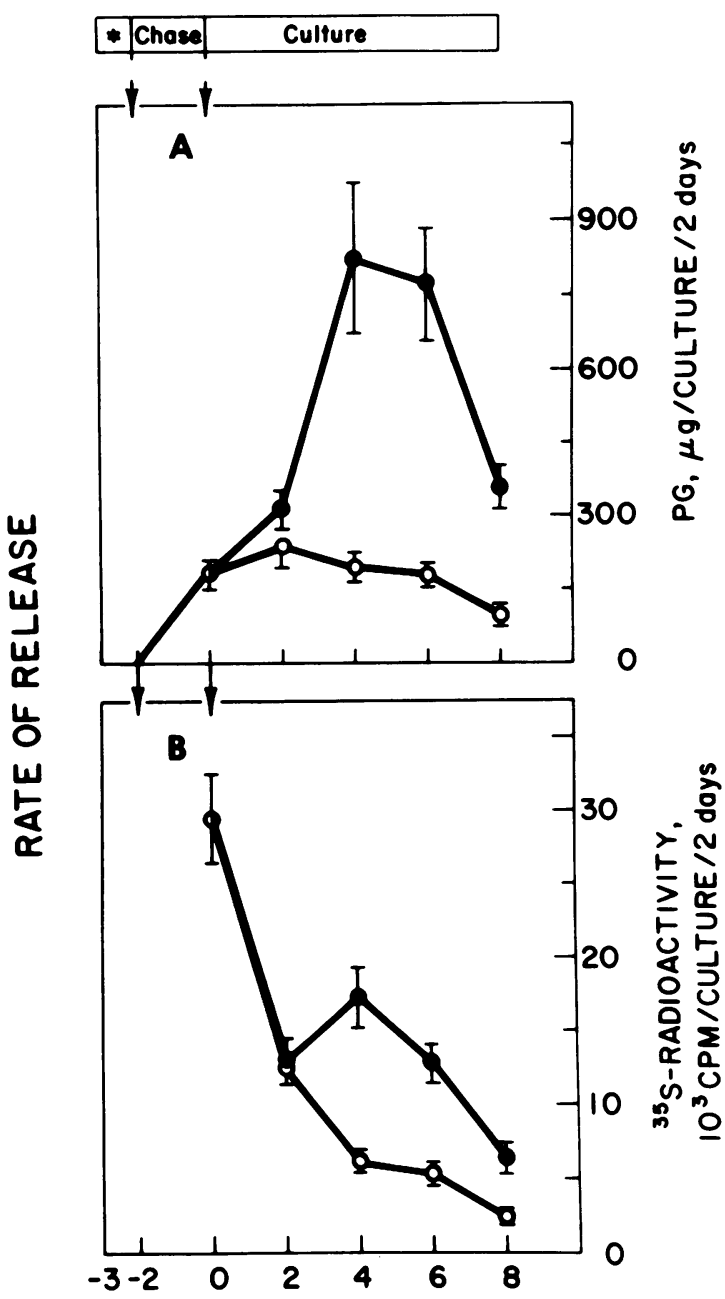

DAYS AFTER ADDITION OF SYNOVIUM

Fig. 4 Degradation of prelabelled cartilage discs by rheumatoid synovial membrane. Cartilage discs incubated for $24 \mathrm{~h}$ in $\mathrm{MgCl}_{2}$-substituted (for $\mathrm{MgSO}_{4}$ ) medium containing $\mathrm{Na}_{2}{ }^{35} \mathrm{SO}_{4}$, were washed with several changes of phosphate-buffered saline and established as individual organ cultures in sulphate-replete medium (first arrow, day -2). After $48 \mathrm{~h}$ (chase), the medium was changed, and minced rheumatoid synovial membrane was applied to one-half of the cultures (second arrow). Cultures were fed at 2-day intervals for an additional 8 days. $O=$ Medium from control cultures; $=$ medium from

cartilage-synovial-membrane cocultures. The rates of $(A)$ proteoglycan and $(B){ }^{35} S$-radioactivity release are shown as means $\pm S E M$ of 5 cultures. 
lag, achieving a maximum within 4-6 days of synovial exposure. The release of ${ }^{35} \mathrm{~S}$-radioactivity (Fig. 4B) was maximal at early stages of culture, declining rapidly thereafter. Again no synovial effect was evident during the first 2 days of exposure, but the breakdown rate was sharply stimulated by day 4 and remained significantly elevated for the duration of the experiment. The accumulation of ${ }^{35} \mathrm{~S}$-labelled products thus generally paralleled the quantitive pattern of proteoglycan release and was significantly enhanced in cultures exposed to synovial membrane.

The suppressive effect of hydrocortisone on cartilage breakdown is illustrated by the experiment depicted in Fig. 5. Breakdown was stimulated in 8 -day cocultures $(\mathrm{C}+\mathrm{S}-\mathrm{H})$, the fractional release of ${ }^{35} \mathrm{~S}$-radioactivity being increased to $47 \%$ of the total (versus $28 \%$ in $\mathrm{C}-\mathrm{H}$ cultures, $\mathrm{p}<0.05$ ). The inclusion of hydrocortisone in the culture medium abolished the response to synovium $(\mathrm{C}+\mathrm{S}+\mathrm{H}$ versus $\mathrm{C}+\mathrm{S}-\mathrm{H}$, $\mathrm{p}<0.05$ ), but produced only minor inhibition of spontaneous breakdown $(\mathrm{C}+\mathrm{H}$ versus $\mathrm{C}-\mathrm{H}$, $0 \cdot 05<\mathrm{p}<0 \cdot 1)$.

Qualitative changes in the ${ }^{35} \mathrm{~S}$-products released into the breakdown pool were sought by gel filtration chromatography of each 2-day medium collection (Fig. 6). In all cultures free and small-molecular

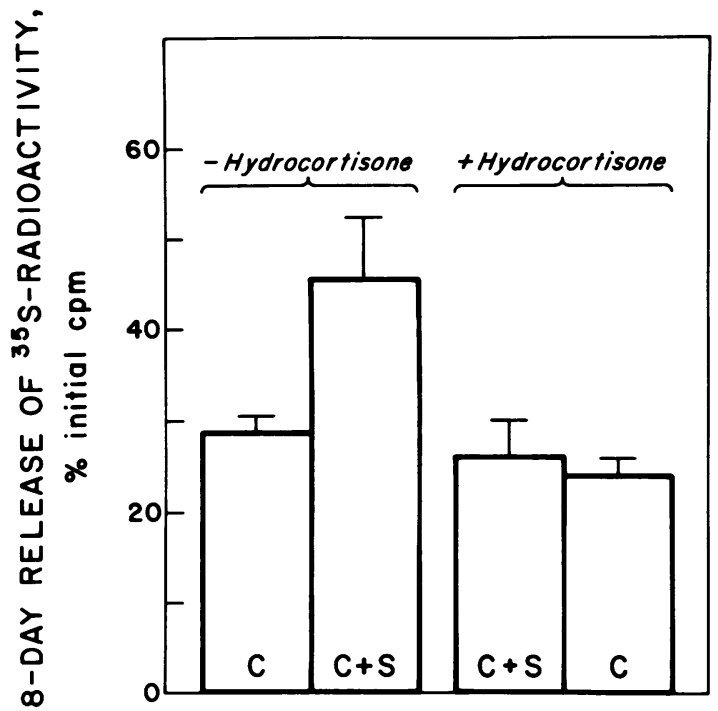

Fig. 5 Effect of hydrocortisone on the breakdown of prelabelled cartilage. ${ }^{35} \mathrm{SO}_{4}{ }^{2-}$-prelabelled and chased cartilage discs were incubated for up to 8 days in the absence or the presence of hydrocortisone $(10 \mu \mathrm{g} \mathrm{ml})$. The cumulative ${ }^{35} S$-radioactivity released into culture medium is expressed as a fraction of the total radioactivity in medium plus disc for each culture. Total ${ }^{35} S$-radioactivity recovered was the same as the initial content per disc $(190400 \mathrm{cpm} \pm S D 8200$, $n=25$ ). $C=$ Cartilage alone, $C+S=$ cartilage plus synovial membrane. Values are means $\pm S E M$ of 5 cultures.

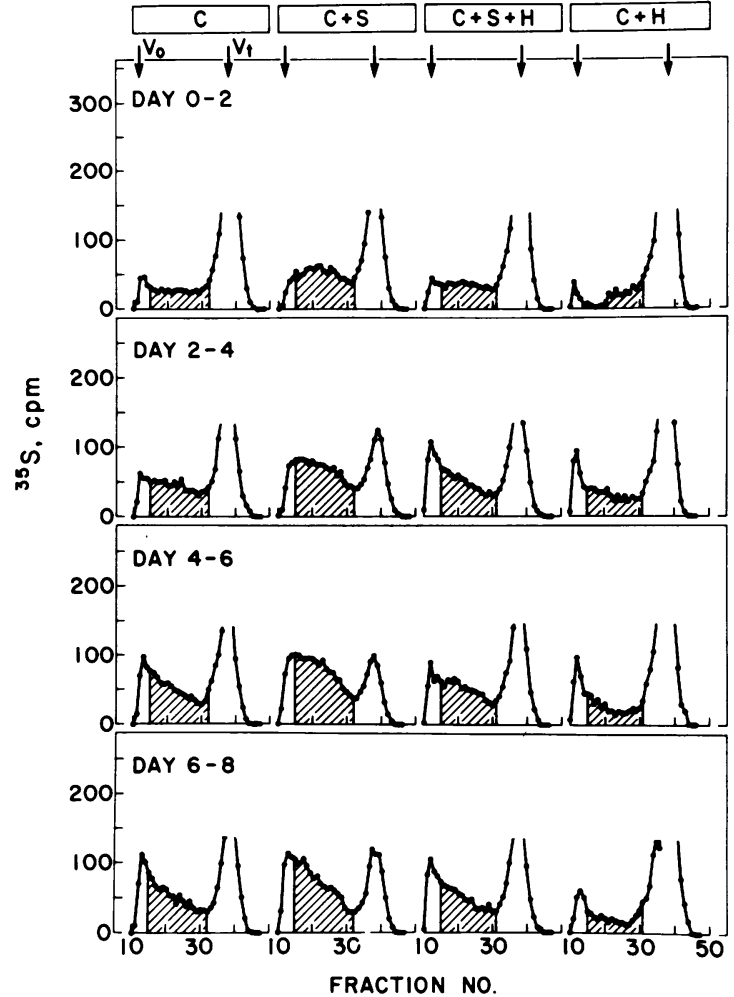

Fig. 6 Gel-filtration chromatography (Sepharose 2B) of ${ }^{35} S$-products released from prelabelled cartilage. Media were obtained at 2-day intervals from cultures containing prelabelled discs which had been incubated in the absence or presence of hydrocortisone $(10 \mu \mathrm{g} \mathrm{ml})$. All profiles have been normalised to the same area under each curve. The shaded areas represent partial degradation products migrating over the $K_{\text {av }}$ range $0 \cdot 12-0 \cdot 74$. Arrows indicate the elution peak positions of blue dextran $2000\left(V_{0}\right)$ and ${ }^{35} \mathrm{SO}_{4}{ }^{2-}(\mathrm{V})$ respectively. $C=$ cartilage alone, $C+S=$ cartilage plus synovial membrane, $C+S+H=$ cartilage plus synovial membrane plus hydrocortisone, $C+H=$ cartilage plus hydrocortisone.

Table 1 The effect of hydrocortisone on release of ${ }^{35} S$-proteoglycan partial degradation products

\begin{tabular}{|c|c|c|c|c|}
\hline \multirow[t]{2}{*}{ Day } & \multicolumn{4}{|c|}{ Fraction of ${ }^{35} S$-radioactivity $(\% \text { total } \mathrm{cpm})^{*}$} \\
\hline & $C$ & $C+S$ & $C+S+H$ & $C+H$ \\
\hline 0 & $16 \cdot 4$ & $36 \cdot 7$ & $23 \cdot 0$ & $10 \cdot 4$ \\
\hline $2-$ & $39 \cdot 6$ & $48 \cdot 4$ & $32 \cdot 6$ & $20 \cdot 9$ \\
\hline 4 & $31 \cdot 1$ & $58 \cdot 1$ & $34 \cdot 3$ & $16 \cdot 2$ \\
\hline $6-8$ & $37 \cdot 1$ & $46 \cdot 2$ & $33 \cdot 8$ & $13 \cdot 0$ \\
\hline Mean & $31 \cdot 1$ & $47 \cdot 4$ & $30 \cdot 9$ & $15 \cdot 1$ \\
\hline \pm SEM & $5 \cdot 2$ & $4 \cdot 4$ & $2 \cdot 7$ & $2 \cdot 3$ \\
\hline
\end{tabular}

${ }^{* 35} \mathrm{~S}$-Radioactivity eluting with $\mathrm{K}_{\mathrm{av}}$ of $0 \cdot 12-0 \cdot 74$, represented by the shaded areas in Fig. 6, is expressed as a percentage of the total in each column run. 
forms to ${ }^{35} \mathrm{SO}_{4}{ }^{2-}$ comprised a large fraction of the total radioactivity. Intermediate-sized breakdown products were prominent in coculture media $(\mathrm{C}+\mathrm{S})$, where they constituted up to $58 \%$ of the total radioactivity (Table 1 ). The addition of hydrocortisone, which quantitatively abolished the synovial response (Fig. 5), reduced the contribution of partial degradation products to $31 \%$ (Table 1 ), tending to restore basal gel filtration profiles (Fig. 6, $\mathrm{C}+\mathrm{S}+\mathrm{H}$ ). Similarly, although there was only minor inhibition of overall breakdown in control cultures (Fig. 5, $\mathrm{C}+\mathrm{H}$ ), the contribution of partial degradation products was sharply reduced by hydrocortisone treatment (Fig. 6 , $\mathrm{C}+\mathrm{H}$ ) to $15 \%$ of the total radioactivity (Table 1 ).

\section{CARTILAGE BREAKDOWN IN SYNOVIAL-} MEMBRANE- CONDITIONED MEDIA (SCM) In cartilage-synovial-membrane cocultures disc erosion is usually noted at the end of 8 days, and proteoglycan release may be increased above basal by as much as 3- to 4-fold. ${ }^{6}$ Changes of this magnitude were also observed when SCM were assayed

Table 2 Cartilage breakdown in synovial-membrane conditioned medium (SCM) and parallel cocultures

\begin{tabular}{|c|c|c|c|c|}
\hline \multirow[b]{3}{*}{ Expt. no. } & \multicolumn{4}{|c|}{ Proteoglycan release, $T / C$ ratio* } \\
\hline & \multicolumn{2}{|c|}{ - Hydrocortisone } & \multicolumn{2}{|c|}{ + Hydrocortisone } \\
\hline & Coculture & SCM assay & Coculturet & SCM assay $\ddagger$ \\
\hline 1 & 3.98 & $1 \cdot 25$ & $3.62(12) \S$ & $1 \cdot 15(40)$ \\
\hline 2 & $3 \cdot 40$ & $2 \cdot 57$ & $2 \cdot 82(24)$ & $1 \cdot 79(50)$ \\
\hline 3 & $2 \cdot 10$ & $2 \cdot 60$ & $2 \cdot 08(2)$ & $2 \cdot 63(-3)$ \\
\hline 4 & $1 \cdot 97$ & $2 \cdot 30$ & $1 \cdot 73(25)$ & $1 \cdot 65(50)$ \\
\hline 5 & $4 \cdot 50$ & $4 \cdot 60$ & $3.56(27)$ & $3.99(17)$ \\
\hline
\end{tabular}

*Treatment/control ratio in each experiment is the mean of 5 replicate cultures. Proteoglycan release ratios in coculture: (cartilage + synovium)/cartilage; in SCM assay: $50 \% \mathrm{SCM}$ /fresh medium, $5 \%$ serum.

tContaining hydrocortisone, $10 \mu \mathrm{g} / \mathrm{ml}$ during coculture.

¥SCM generated in hydrocortisone-containing medium, $10 \mu \mathrm{g} / \mathrm{ml}$.

$\S$ Percent inhibition, calculated as the difference in increment above basal proteoglycan release in $(+)$ hydrocortisone versus $(-)$ hydrocortisone cultures.

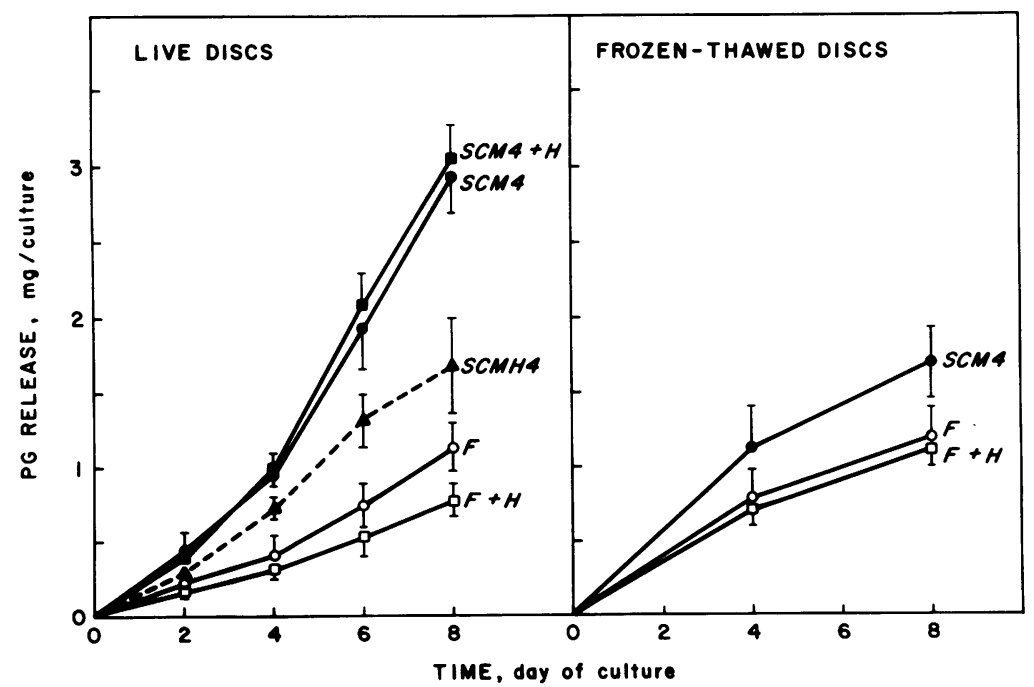

Fig. 7 Cartilage breakdown in response to synovial membrane conditioned media. Conditioned media were pooled during 4 days' incubation of synovial explants cultivated either in the absence (SCM4) or presence (SCMH4) of hydrocortisone, $5 \mathrm{\mu g} / \mathrm{ml}$. All SCM were diluted 1:1 with fresh serum-free medium at the time of assay, and were compared with complete fresh medium $(F)$ containing the same concentration (5\%) of serum. Assay for chondrocyte-dependent breakdown was made by comparing proteoglycan release from living (left) versus repeatedly frozen-thawed discs (right). Assay cultures indicated as $+H(\square, \square)$ were made in the presence of added hydrocortisone $(2 \cdot 5 \mu \mathrm{g} / \mathrm{ml})$. Values are means $\pm S E M$ of 5 cultures. 
separately (Table 2), and the values for degradative activity generally corresponded to those noted in parallel cocultures. The inhibition of breakdown by hydrocortisone, however, corresponded less well in the 2 assay systems. In several experiments (Table 2 , nos. $1,2,4)$, hydrocortisone treatment of synovial explants during the conditioning phase inhibited the capacity of such media to stimulate proteoglycan breakdown to a greater extent than did hydrocortisone treatment of the parallel cocultures.

Because the conditioned media generated by such steroid-treated synovial membranes may contain sufficient hydrocortisone to inhibit the subsequent cartilage assay, experiments were made to compare the degradative responses to SCM either generated in the presence of hydrocortisone or assayed in the presence of added hydrocortisone. The experiment in Fig. 7 demonstrates a $21 / 2$-fold stimulation of cartilage breakdown by 4-day conditioned medium (SCM4), which was evident only in living cartilages. This response was unaffected by the addition of hydrocortisone directly to the assay cultures $(\mathrm{SCM} 4+\mathrm{H})$, although there was modest suppression in control cultures $(\mathrm{F}+\mathrm{H})$. On the other hand hydrocortisone treatment during the conditioning phase of synovial culture (SCMH4) reduced the potency of that medium by approximately one-half.

\section{Discussion}

We have previously shown that proteoglycan released into the culture medium - at least $70 \%$ arising from matrix breakdown - serves as a useful index of cartilage degradation. ${ }^{6}$ In the present experiments proteoglycan release was measured under conditions in which neither the quantity of cartilage nor the amount of synovium was limiting; rather, both were present in excess. The occasional error in synovial delivery, even if it were to exceed the coefficient of variation of the method (i.e., $20 \%$ ), still provides a synovial dose that is adequate to produce maximal proteoglycan breakdown (Fig. 1). Moreover, Fig. 5 illustrates the relative abundance of cartilage substrate, for a large fraction of the radioactivity originally present in prelabelled discs generally still remains even after 8 days of coculture. The absence of additional proteoglycan release at doses of synovial protein $>200 \mu \mathrm{mol}$ of amino acid (Fig.1) indicates a maximum degradative effort - either maximal chondrocyte responsiveness, maximal release of synovial factors, or both. Restrictions imposed by the geometry of the culture system, i.e., tissue contact or limitation of diffusion at high synovial dose, may interfere with tissue interactions, thereby limiting the degradative response, even though a component of breakdown is known to occur independently of such direct contact. ${ }^{4}{ }^{5} 7$
Cartilage breakdown is inhibited by hydrocortisone in a dose-dependent fashion, with synovialmembrane-induced resorption seemingly more sensitive than spontaneous degradation (Fig. 2). We have previously shown that these 2 components of breakdown can be dissociated, and that the sensitivity of autolysis to hydrocortisone inhibition is proportional to its basal rate. ${ }^{8}$ Thus, if that rate is low, there may be little hydrocortisone response in control cultures despite a major reduction of synovialmembrane-induced breakdown in the parallel cocultures. Synovial membrane itself releases little proteoglycan irrespective of the presence of hydrocortisone. This confirms the observation ${ }^{6}$ that synovial membrane is not a significant source of the high-charge-density polyanions (i.e., sulphated glycosaminoglycans) measured by the alcian blue assay, ${ }^{12}$ and that the latter method thus preferentially distinguishes products originating in cartilage.

From a consideration of the proteoglycan stoichiometry it is evident that synovial membrane inhibits net proteoglycan synthesis, as $\mathrm{we}^{6}$ and others $^{14}$ have reported, and that pharmacological doses of hydrocortisone produce little additional inhibition (Fig. 3). Although the drug does inhibit synthesis in control cultures (Fig. 3, C+H), its major effect is to suppress breakdown. At times this effect can be striking, both in the suppression of spontaneous and of synovial-membrane-induced breakdown (Figs. 3 and 6). Hydrocortisone not only reduces the total quantity of breakdown products shed into culture medium but also alters their molecular sizedistribution, so that a lesser fraction appears as partial degradation products (Fig. 6). We have presented evidence that such intermediate-sized molecules differ largely on the basis of their protein-core lengths, ${ }^{15}$ and therefore represent a spectrum of proteinase-digested proteoglycan fragments. A number of potential enzyme candidates, active at neutral or acid $\mathrm{pH}$, have been described in synovial membrane and cartilage, ${ }^{16-22}$ one or more of which may be implicated in this process.

The loss of synovial protein through autolysis could be prevented by the inclusion of hydrocotisone at sufficiently high concentration in culture medium (Fig. 2). This was paralleled by a reduction of cartilage proteoglycan release (Fig. 2) and of the contribution of proteolytic cleavage products (Fig. 6). Both are consistent with the known effect of hydrocortisone at pharmacological doses to stabilise cellular membranes, thereby inhibiting endocytosis and lysosomal enzyme discharge. ${ }^{23-25}$ Although inhibition of proteoglycan breakdown thus accompanies a reduction in endogenous proteinase activity, hydrocortisone stabilisation of cellular and lysosomal membranes may represent only a part of the corticosteroid response. 
In actively resorbing cultures the steroid effect is clearly demonstrable within 4 days. ${ }^{9}$ However, the data in Table 2 and Fig. 7 indicate that hydrocortisone may also inhibit the production of synovial factors which stimulate the chondrocyte-dependent breakdown of cartilage. These findings are in accord with similar experiments recently reported by Saklatvala ${ }^{26}$ and suggest that the degradative response can be significantly blocked by steroid at a critical early phase of the interaction between synovial membrane and cartilage. Such blockade of induction' may be more immediately related to early transcriptional or protein-synthetic events, ${ }^{27}$ quite apart from any membrane stabilising effect of hydrocortisone. Moreover, we have demonstrated that inhibition of collagen breakdown in this system is exquisitely sensitive to hydrocortisone, even in the face of persisting proteoglycan breakdown. ${ }^{28}$ This dissociation is consistent with different rate-limiting steps or enzymatic control points for the 2 catabolic processes, perhaps mediated by different primary or amplifier cell populations. The latter possibility is supported by the implication of mononuclear cell factors in the elaboration of synovial collagenase ${ }^{2930}$ and the known sensitivity of such cells to glucocorticoids.

We thank Patricia Mark and Sonya Shortkroff for expert technical assistance and Terri Sayles for preparation of the manuscript. Supported by National Institutes of Health grant numbers AM 18268 and AM 23063.

\section{References}

1 Barrett A J. The enzymic degradation of cartilage matrix. In: Burleigh P M C, Poole A R, eds. Dynamics of Connective Tissue Macromolecules. Amsterdam: North Holland, 1975; 189-215.

2 Kobayashi E D, Ziff M. Electron microscopic studies of the cartilage-pannus junction in rheumatoid arthritis. Arthritis Rheum 1977; 18: 475-83.

3 Harris E D, Glauert A M, Murley A H G. Intracellular collagen fibers at the pannus-cartilage junction in rheumatoid arthritis. Arthritis Rheum 1977; 20: 657-65.

4 Fell H B, Jubb R W. The effect of synovial tissue on the breakdown of articular cartilage in organ culture. Arthritis Rheum 1979; 20: 1359-71

5 Mitchell N S, Shepard N. Changes in proteoglycan and collagen in cartilage in rheumatoid arthritis. J Bone Joint Surg 1978; 60A: 349-54.

6 Steinberg J, Sledge C B, Noble J, Stirrat C R. A tissue-culture model of cartilage breakdown in rheumatoid arthritis. Quantitative aspects of proteoglycan release. Biochem J 1979; 180: 403-12.

7 Dingle J T, Saklatvala J, Hembry R, Tyler J, Fell H B, Jubb $R$. A cartilage catabolic factor from synovium Biochem $J$ 1979; 184: 177-80.

8 Steinberg J, Tsukamoto S, Sledge C B. A tissue culture model of cartilage breakdown in rheumatoid arthritis. III. Effects of antirheumatic drugs. Arthritis Rheum 1979; 22: 877-85.

9 Sledge C B, Steinberg J J. Co-cultivation models of joint destruction. In: Dingle J T, Gordon J L. eds. Cellular
Interactions. Amsterdam: Elsevier/North-Holland Biomedical Press, 1981: 263-80.

10 Sajdera S W, Hascall V C. Proteinpolysaccharide complex from bovine nasal cartilage. A comparison of low and high shear extraction procedures. J Biol Chem 1969; 244: 77-87.

11 Bailey J L. Techniques in Protein Chemistry. Amsterdam: Elsevier, 1967: 79.

12 Whiteman $P$. The quantitative measurement of alcian blue-glycosaminoglycan complexes. Biochem $J$ 1973; 131: 343-50.

13 Documenta Geigy Scientific Tables. Ed. K Diem. Ardsley N Y: Geigy Chemical Corporation, 1962.

14 Jubb R W, Fell H B. The effect of synovial tissue on the synthesis of proteoglycan by the articular cartilage of young pigs. Arthritis Rheum 1980; 23: 545-55.

15 Steinberg J J, Sledge C B. A tissue culture model of rheumatoid arthritis. Role of proteoglycan-core proteolysis in cartilage breakdown. Trans Orthopaed Res Soc 1982; 7: 134.

16 Ali S Y, Evans L. Enzymic degradation of cartilage in osteoarthritis. Fed Proc 1973; 32: 1494-8.

17 Sapolsky A I, Howell D S, Woessner J F Jr. Neutral proteases and cathespin $\mathrm{D}$ in human articular cartilage. $J$ Clin Invest 1974; 53: 1044-53.

18 Sapolsky A I, Keiser H, Howell D S, Woessner J F Jr. Metalloproteases of human articular cartilage that digest cartilage proteoglycan at neutral and acid $\mathrm{pH}$. J Clin Invest 1976; 58: 1030-41.

19 Janoff A, Feinstein G, Malemud C J, Elias J M. Degradation of cartilage proteoglycan by human leukocyte granule neutral proteases-a model of joint injury. I. J Clin Invest 1976; 57: 615-24.

20 Keiser H, Greenwald R A, Feinstein G, Janoff A. Degradation of cartilage proteoglycan by human leukocyte granule neutral proteases - a model of joint injury. II. J Clin Invest 1976; 57: 625-32.

21 Roughley P J, Barrett A J. The degradation of cartilage proteoglycans by tissue proteinases. Proteogylcan structure and its susceptibility to proteolysis. Biochem $J$ 1977; 167: 629-37.

22 Dingle J T. Recent studies on the control of joint damage: the contribution of the Strangeways Research Laboratory. Ann Rheum Dis 1979; 38: 201-14.

23 Weissmann G. Lysosomal mechanisms of tissue injury in arthritis. N Engl J Med 1972; 286: 141-7.

24 Weissmann $\mathrm{G}$. The effects of steroids and drugs on lysosomes. In: Dingle J T, Fell $\mathrm{H} \mathrm{B}$, eds. Lysosomes in Biology and Pathology. Amsterdam: North-Holland, 1969: 1: 276-95.

25 Thomas D P P. Lysosomal enzymes in experimental and rheumatoid arthritis. In: Dingle J T, Fell H B, eds. Lysosomes in Biology and Pathology. Amsterdam: North-Holland, 1969: 2: $87-110$.

26 Saklatvala J. Characterization of catabolin, the major product of pig synovial tissue that induces resorption of cartilage proteoglycan in-vitro. Biochem J 1981; 199: 705-14.

27 Yamamoto K R, Alberts B M. Steroid receptors: elements for modulation of eukaryotic transcription. Annu Rev Biochem 1976; 45: 721-46.

28 Steinberg J, Kincaid S B, Tsukamoto S, Sledge C B Differential inhibition by cortisol of collagen and proteoglycan breakdown in a tissue culture model of rheumatoid arthritis. Orthopaed Trans 1980; 4: 233-4.

29 Dayer J-M, Russell R G G, Krane S M. Collagenase production by rheumatoid synovial cells: stimulation by a human lymphocyte factor. Science 1977; 195: 181-3.

30 Dayer J-M, Breard J, Chess L, Krane S M. Participation of monocyte-macrophages and lymphocytes in the production of a factor that stimulates collagenase and prostaglandin release by rheumatoid synovial cells. J Clin Invest 1979; 64: 1386-92. 